\title{
Escalas para prevenção de lesão por pressão em unidades de terapia intensiva: revisão integrativa
}

\author{
Pressure injury prevention scales in intensive care units: an integrative review
}

\section{Como citar este artigo:}

Almeida ILS, Garces TS, Oliveira GYM, Moreira TMM. Pressure injury prevention scales in intensive care units: an integrative review. Rev Rene. 2020;21:e42053. DOI: https://doi.org/10.15253/2175-6783.20202142053

\author{
(DÍtalo Lennon Sales de Almeida ${ }^{1}$ \\ DThiago Santos Garces ${ }^{1}$ \\ (D)Glória Yanne Martins de Oliveira ${ }^{1}$ \\ (DThereza Maria Magalhães Moreira ${ }^{1}$
}

\begin{abstract}
RESUMO
Objetivo: descrever os elementos constitutivos do cuidado de enfermagem presentes nas escalas de avaliação do risco de lesão por pressão usadas em unidades de terapia intensiva. Métodos: trata-se de uma revisão integrativa da literatura a partir do LILACS, MEDLINE, SCIELO e BDENF. Os descritores utilizados para a busca foram Pressure Ulcer; Decubitus Ulcer; Prevention and Control; Prevention; Intensive Care Units. A amostra final foi constituída por 13 artigos científicos. Resultados: a escala mais utilizada entre os estudos analisados foi Braden. Os elementos constitutivos evidenciados foram avaliação estruturada do risco, avaliação da pele e tecidos, cuidados preventivos com a pele, nutrição, reposicionamento no leito, superfícies de apoio e cuidados com dispositivos médicos. Conclusão: a prevenção de lesões relacionadas às incontinências, avaliação nutricional, intervenções nutricionais com o objetivo de prevenir lesões e os cuidados relacionados a dispositivos médicos são elementos constitutivos do cuidado de enfermagem pouco explorados ou ausentes nas escalas avaliadas.

Descritores: Lesão por Pressão; Prevenção \& Controle; Unidade de Terapia Intensiva; Enfermagem.
\end{abstract}

\section{ABSTRACT}

Objective: to describe the constituent elements of nursing care present in the pressure injury risk assessment scales used in intensive care units. Methods: this is an integrative literature review based on LILACS, MEDLINE, SCIELO and BDENF. The descriptors used for the search were Pressure Ulcer; Decubitus Ulcer; Prevention and control; Prevention; Intensive Care Units. The final sample consisted of 13 scientific articles. Results: the Braden scale was the most used scale among the analyzed studies. The constituent elements highlighted were structured risk assessment, skin and tissue assessment, preventive skin care, nutrition, repositioning in bed, support surfaces, and care with medical device. Conclusion: the prevention of incontinence-related injuries, nutritional assessment, nutritional interventions aimed at preventing injuries and care with medical devices are constituent elements of nursing care still poorly explored or absent in the evaluated scales.

Descriptors: Pressure Ulcer; Prevention \& Control; Intensive Care Units; Nursing. 


\section{Introdução}

A lesão por pressão é definida pelo National Pressure Ulcer Advsory Panel (NPUAP) como um dano na pele ou demais tecidos, ocorrendo geralmente sobre uma superfície óssea ou relacionada ao uso de dispositivos para cuidado em saúde ou outros artefatos. É altamente incidente em ambientes hospitalares, principalmente em unidades de internação de pacientes criticamente doentes, como as unidades de terapia intensiva ${ }^{(1-2)}$. A prevenção das lesões representa importante desafio aos profissionais da prática clínica em terapia intensiva.

Entre as principais medidas preventivas estão o uso de escalas para detectar o risco e a suscetibilidade do paciente ao acometimento por tal evento. Tais ferramentas subsidiam a criação de um plano de cuidados pertinente, que evita ou diminui o desenvolvimento das lesões ${ }^{(3-4)}$. Quando se fala em cuidado, o enfermeiro é o profissional que estabelece maior contato com os pacientes dentro da unidade de terapia intensiva, visto que a manutenção da integridade da pele está incluída no plano de cuidados da enfermagem e deve contemplar o risco de desenvolvimento de lesões para atuar na prevenção e em situações de integridade da pele prejudicada, tratando- $\mathrm{a}^{(5)}$.

Para a enfermagem em terapia intensiva, é consenso que a manutenção da integridade da pele é mais viável quando realizada com técnicas e tecnologias simples e econômicas, como escalas que medem o risco do surgimento de lesões em pacientes. Nessa perspectiva, tem-se buscado formas sistematizadas de medição do risco que avaliam as diversas condições clínicas envolvidas no surgimento de lesões de modo efetivo e com enfoque na diminuição dos desfechos mais graves associados, como infecções, desfecho cirúrgico desfavorável, tempo de internação hospitalar e mortalidade ${ }^{(6)}$.

Nesse sentido, o olhar sobre essas escalas é fundamental, pois possibilita o desenvolvimento de uma abordagem estruturada, individualizada e sistematizada para superar os desafios encontrados e am- pliar os aspectos facilitadores naquilo que diz respeito ao cuidado de enfermagem direcionado à prevenção de lesões por pressão em terapia intensiva, tendo em vista que a qualidade do cuidado de enfermagem está intrinsecamente associada a uma menor incidência de lesões nas instituições que prestam assistência à saú$\mathrm{de}^{(5)}$.

Com base no exposto, propomos realizar uma revisão integrativa com objetivo de descrever os elementos constitutivos do cuidado de enfermagem presentes nas escalas de avaliação do risco de lesão por pressão usadas em unidades de terapia intensiva. Nesse contexto, justifica-se analisar as evidências disponíveis acerca das escalas que vêm sendo utilizadas em unidades de terapia intensiva e quais elementos constitutivos do cuidado são permeados pela sua avaliação. Essa análise é relevante, tendo em vista que essas escalas são usadas para subsidiar cuidados preventivos e boas práticas de enfermagem nos cuidados intensivos, logo podem influenciar na qualidade dos cuidados destinados aos pacientes diminuindo a exposição a riscos de ocorrência de eventos adversos, tornando a assistência de enfermagem mais segura.

\section{Métodos}

Trata-se de uma revisão integrativa, que buscou as principais evidências disponíveis sobre o objeto de estudo, sendo realizada em seis etapas, assim nomeadas: identificação do tema; busca na literatura (seleção de critérios de inclusão e exclusão); categorização dos estudos; avaliação dos estudos; interpretação dos resultados; síntese do conhecimento ${ }^{(7-8)}$.

A busca pareada foi realizada de novembro a dezembro de 2018. Incluíram-se os estudos primários quantitativos disponíveis na íntegra; em língua inglesa, portuguesa ou espanhola; publicados entre 2007 e 2018, período selecionado considerando que as evidências sobre lesões por pressão dos últimos doze anos são, atualmente, as mais utilizadas; cabe ressaltar também, com relação ao marco temporal, que esse período coincide com a publicação da versão adapta- 
da e validada para o português da escala de Braden Q no ano de 2007. Incluíram-se estudos desenvolvidos em ambiente de terapia intensiva adulta e pediátrica. Adotaram-se como critério de exclusão estudos que não trouxessem em seu resultado evidências claras sobre uso das escalas e elementos constitutivos que mais impactavam na prevenção de lesão por pressão.

A estruturação da questão norteadora da revisão integrativa envolveu a estratégia $\mathrm{PICO}^{(9)}[\mathrm{P}(\mathrm{Popu}-$ lação)= indivíduos internados em unidade de terapia intensiva; I (intervenção): escalas sobre lesão por pressão; C (comparação): não tem; O (resultados): elementos constitutivos]. A pergunta norteadora para a condução da revisão integrativa foi: quais elementos constitutivos do cuidado de enfermagem são embasados no uso das escalas de avaliação do risco para lesão por pressão em unidades de terapia intensiva?

Os descritores foram selecionados com base na estratégia PICO. Adicionou-se o operador booleano OR entre os sinônimos e AND entre os termos diferentes, formando a chave de busca: Pressure Ulcer OR Decubitus Ulcer AND Prevention and Control OR prevention AND Intensive Care Units.

A escolha das bases de dados considerou sua abrangência e afinidade com o tema. A busca eletrônica da literatura foi realizada a partir das bases de dados LILACS (Literatura Latino-Americana e do Caribe em Ciências da Saúde), MEDLINE (Sistema Online de Busca e Análise de Literatura Médica) por meio da interface Pubmed e BDENF (Base de Dados em Enfermagem) e da biblioteca do SCIELO (Scientific Electronic Library Online). Nas bases de dados, selecionaram-se os filtros de busca referentes às publicações disponíveis na íntegra; em língua portuguesa, inglesa ou espanhola; anos de publicação; tipo de publicação. A busca e a seleção dos artigos incluídos na revisão foram realizadas por dois revisores de forma independente para validação da busca e seleção.

O processo de seleção dos estudos foi conduzido por meio do protocolo Preferred Reporting Items for Systematic reviews and Meta-Analyses (PRISMA). 0 fluxograma (Figura 1) mostra o caminho percorrido para a seleção das publicações.

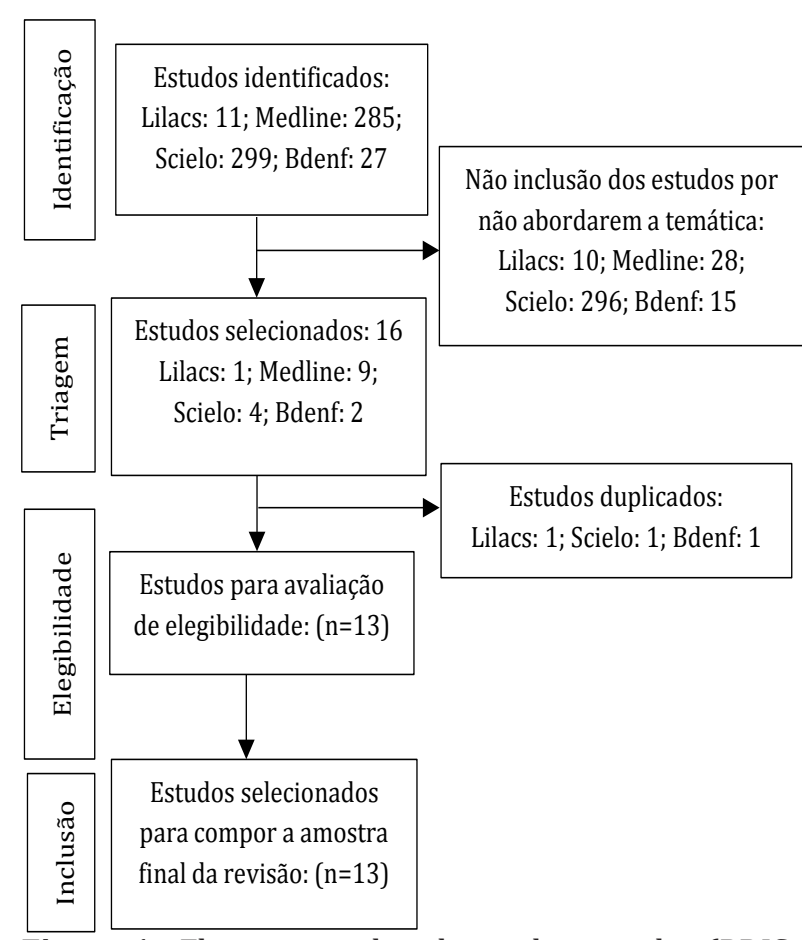

Figura 1 - Fluxograma de seleção dos estudos (PRISMA). Fortaleza, CE, Brasil, 2019

Os estudos elegíveis foram lidos na íntegra pelos dois pesquisadores, os quais extraíram os dados previamente definidos com uso de um instrumento criado pelos próprios autores. 0 local e ano de realização do estudo, o delineamento metodológico adotado, a escala aplicada e os elementos constitutivos dela foram as informações extraídas. Cada pesquisador chegou a uma lista de estudos primários. As duas listas foram comparadas e consolidadas em uma única lista. Em caso de divergência sobre a inserção ou não de algum artigo na lista final, um terceiro pesquisador ligado ao tema foi consultado. Foram selecionados e analisados 13 estudos para compor o corpus desta revisão. A partir da leitura e análise crítica, procedeu-se à discussão e intepretação dos resultados obtidos com a apresentação das evidências encontradas. A análise dos dados coletados nos estudos se deu por método descritivo.

As nomenclaturas usadas pelos autores das pesquisas em análises foram adotadas para definir os desenhos dos estudos primários que fizeram parte desta pesquisa, quando não foi possível identificar 
de forma clara nos artigos. A força da evidência foi definida pelas diferentes questões de pesquisa estabelecidas por Fineout-Overholt e Stillwell. Assim, dependendo da questão do estudo primário (significado; Prognóstico/Predição ou Etiologia; intervenção/ tratamento ou diagnóstico/teste de Diagnóstico) ${ }^{(10)}$.

Os estudos foram analisados para que fossem realizadas a sua descrição e avaliação do seguimento dos elementos constitutivos propostos pelo guideline Prevention and Treatment of Pressure Ulcers: Quick Reference Guide, criado pela National Pressure Ulcer Advisory Panel (NPUAP). A NPUAP indica em sua publicação como principais recomendações para a prevenção de lesões por pressão a avaliação de risco, avaliação da pele e dos tecidos, cuidados preventivos com a pele, terapias emergentes para prevenção de lesões por pressão, nutrição, reposicionamento e mobilização no leito, superfícies de apoio e cuidados com dispositivos para cuidado em saúde. Cada um dos aspectos será discutido e apontada sua presença nas referências analisadas ${ }^{(6)}$.

\section{Resultados}

Os dados sintetizados nos estudos foram organizados em uma matriz de dados de exibição. A matriz contém informações referentes ao tipo de escala e elementos constitutivos (Figura 2).

\begin{tabular}{|c|c|}
\hline Escalas & Elementos constitutivos \\
\hline $\begin{array}{l}\text { Escala para avaliação de } \\
\text { risco (Norton) }{ }^{(11)}\end{array}$ & $\begin{array}{l}\text { Documentação detalhada; Qualidade do registro; Reavaliação periódica da pele; Reposicionamento; Aplicação de cre- } \\
\text { me hidratante; Colchão de redução da pressão. }\end{array}$ \\
\hline $\begin{array}{l}\text { Escala para avaliação de } \\
\text { risco (Waterlow) }\end{array}$ & $\begin{array}{l}\text { reinamentos para qualidade na avaliação da pele; Uso de dispositivos para alívio da pressão; Cuidados com disposi- } \\
\text { ivos médicos. }\end{array}$ \\
\hline $\begin{array}{l}\text { Escala para avaliação de } \\
\text { risco (Braden } Q)^{(13)}\end{array}$ & $\begin{array}{l}\text { valiação da pele periódica e registro; Uso de colchão para redistribuição de pressão; Reposicionamento; Nutrição } \\
\text { avaliação da proteína dietética); Cuidados com dispositivos médicos. }\end{array}$ \\
\hline $\begin{array}{l}\text { Escala para avaliação de } \\
\text { risco }(\text { Gosnell) })^{(14)}\end{array}$ & $\begin{array}{l}\text { Avaliação diária da pele; Uso de roupa de algodão; Lavagem e secagem da pele (evitar excesso de umidade); Pressão } \\
\text { manipulada com dispositivos; Reposicionamento (4 horas); Uso de ácidos graxos oxigenados; Colchão de espuma visco- } \\
\text {-elástico. }\end{array}$ \\
\hline $\begin{array}{l}\text { Escala para avaliação de } \\
\text { risco (Braden) }{ }^{(15)}\end{array}$ & $\begin{array}{l}\text { Escala para reposicionamento (3 horas); Cuidados com a umidade da pele; Avaliação nutricional; Uso de filme transpa- } \\
\text { rente; Uso de creme barreira (Cavilon); Uso de colchão de espuma piramidal. }\end{array}$ \\
\hline $\begin{array}{l}\text { Protocolo para prevenção } \\
\text { de lesão por pressão em } \\
\text { pós-operatório de cirur- } \\
\text { gia cardíaca }^{(16)}\end{array}$ & Iso de colchão de fluido de ar para diminuição de fricção e cisalhamento. \\
\hline $\begin{array}{l}\text { Escala para avaliação de } \\
\text { risco (Braden) }{ }^{(17)}\end{array}$ & $\begin{array}{l}\text { Criação de uma equipe de enfermagem específica para avaliação da pele e feridas; Educação permanente; Documenta- } \\
\text { ção de qualidade; Rondas semanais; Uso de dispositivos para posicionamento; Produtos de cuidado com a pele (hidra- } \\
\text { tantes, AGE, creme barreira). }\end{array}$ \\
\hline $\begin{array}{l}\text { Escala para avaliação de } \\
\text { risco (Braden) }{ }^{(18)}\end{array}$ & $\begin{array}{l}\text { Avaliação diária da pele; Mudança de decúbito (2 horas); Uso de colchão pneumático; Solicitar suporte/avaliação nu- } \\
\text { tricional; Uso de dispositivos de alívio de pressão; Minimizar exposição da pele à umidade; Sabonete Glicerinado: não } \\
\text { altera o pH da pele e não causa ressecamento; Evitar água quente; Excessiva fricção durante o banho; Uso de protetor } \\
\text { para a pele (ácidos graxos essenciais - AGE, Cavilon); Não massagear áreas com hiperemia; Evitar massagear áreas de } \\
\text { proeminências ósseas. }\end{array}$ \\
\hline $\begin{array}{l}\text { Escala para avaliação de } \\
\text { risco (Braden Q) }{ }^{(19)}\end{array}$ & $\begin{array}{l}\text { valiação da pele; Cuidados com a pele; Cuidados com o paciente indiretamente relacionados à pele (controle da dor, } \\
\text { utrição, hidratação); Produtos relacionados à pressão; Envolvimento paciente / familiar. }\end{array}$ \\
\hline $\begin{array}{l}\text { Escala de avaliação nutri- } \\
\text { cional }^{(20)}\end{array}$ & valiar risco nutricional e implementar melhores formas de suplementação de nutrientes na dieta dos pacientes. \\
\hline $\begin{array}{l}\text { Escala para avaliação de } \\
\text { risco (Braden) }{ }^{(21)}\end{array}$ & $\begin{array}{l}\text { eposicionamento fracionado e observação dos parâmetros do paciente com instabilidade hemodinâmica; Cuidados } \\
\text { om dispositivos médicos. }\end{array}$ \\
\hline $\begin{array}{l}\text { Escala para avaliação de } \\
\text { risco (Braden) }{ }^{(22)}\end{array}$ & ispositivos para alívio da pressão; Curativos de silicone; Equipe de avaliação da qualidade. \\
\hline $\begin{array}{l}\text { Escala para avaliação de } \\
\text { risco (Braden) }{ }^{(23)}\end{array}$ & $\begin{array}{l}\text { Uso de drogas vasoativas, condições da pele e uso de ventilação mecânica invasiva; Avaliação de risco de lesão por } \\
\text { pressão por meio da pontuação da escala de Braden; Ocorrência e localização de lesões por pressão. }\end{array}$ \\
\hline
\end{tabular}

Figura 2 - Caracterização da produção científica sobre as escalas de prevenção para lesão por pressão em unidades de terapia intensiva. Fortaleza, CE, Brasil, 2019 
Entre os estudos selecionados, seis $(46,0 \%)$ foram desenvolvidos nos Estados Unidos ${ }^{(13,16-17,19,21-22)}$, três $(23,6 \%)$ são brasileiros ${ }^{(15,18,23)}$, um $(7,6,3 \%)$ é espanhol $^{(14)}$, um $(7,6 \%)$ é de Portugal ${ }^{(11)}$, um $(7,6 \%)$ é da Austrália $^{(12)}$ e um $(7,6 \%)$ é do México ${ }^{(20)}$.

No tocante ao ano de publicação, um $(7,6 \%)$ artigo foi publicado em $2007^{(11)}$, três $(22,8 \%)$ em $2008^{(12-14)}$, um $(7,6 \%)$ em $2010^{(15)}$, um $(7,6 \%)$ em $2011^{(16)}$, um $(7,6 \%)$ em $2012^{(17-18)}$, um $(7,6 \%)$ em $2013^{(19)}$, dois $(15,2 \%)$ em $2015^{(20-21)}$, um $(7,6 \%)$ em $2016^{(22)}$ e um $(7,6 \%)$ em $2018^{(23)}$. Quanto ao delineamento dos estudos, oito (61\%) eram pesquisas des$\operatorname{critivas}^{(11,13,15-18,20-21)}$, dois $(15,3 \%)$ eram quase experimentais $^{(12,22)}$ e três $(23,7 \%)$ eram estudos transversais analíticos $^{(14,19,23) \text {. }}$

Quanto aos tipos de unidades, seis (46\%) eram do tipo geral adulto ${ }^{(11,12,15,18,20,23)}$, três $(23,7 \%)$ cardiológicas (coronarianas) $)^{(14,16,21)}$, duas $(15,1 \%)$ do tipo geral pediátrica ${ }^{(13,19)}$, uma $(7,6 \%)$ do tipo neurológica adulto $^{(17)}$ e uma $(7,6 \%)$ do tipo clínico/cirúrgica adulto $^{(22)}$

No que se refere à abordagem dos estudos, todos eram estudos quantitativos. Em análise do nível de evidência dos artigos, observou-se que oito $(60,8$ \%) possuíam nível IIII ${ }^{(12-13,16-17,19-22)}$ e cinco $(39,2 \%)$ possuíam nível I[ ${ }^{(11,14-15,18,23)}$.

Todas as referências analisadas apontaram o uso de algum instrumento para a avaliação de risco. Os instrumentos citados foram a escala de Bra$\operatorname{den}^{(15,17-18,21-23)}$ em seis estudos e sua versão adaptada para pediatria (Braden Q) ${ }^{(13,19)}$ em dois estudos, a escala de Norton $^{(11)}$, a escala de Waterlow ${ }^{(12)}$ e a escala de Gosnell ${ }^{(14)}$ em um estudo cada. Apenas duas referências não fizeram o uso de escalas validadas para avaliar especificamente o risco de desenvolvimento de lesão por pressão ${ }^{(16,20)}$.

Os dois estudos desenvolvidos em unidades de terapia intensiva pediátricas utilizaram a versão Braden Q para avaliação de risco para lesão por pressão. Trata-se da adaptação do instrumento indicada para pacientes de um mês de vida até escolares, sendo a versão de escolha para aplicação em unidades de terapia intensiva pediátricas.

\section{Discussão}

A limitação do estudo está no fato de que foram avaliadas somente evidências geradas em pesquisas realizadas em unidades de terapia intensiva, o que restringiu uma avalição mais ampla dos elementos constitutivos que compõem as escalas utilizadas para prevenção de lesões no ambiente hospitalar de forma geral.

A análise do uso das escalas e seus elementos constitutivos junto do cuidado de enfermagem contribui para a explanação do que vem sendo utilizado como tecnologia do cuidado para a prevenção de lesões. Esse tipo de investigação corrobora com o paradigma de redução dos riscos a que os pacientes estão expostos durante sua internação em ambiente hospitalar. A enfermagem é um componente fundamental no sistema de prevenção de eventos adversos, como a lesão por pressão, que tem relação direta com os cuidados diretamente ofertados ${ }^{(4,6)}$.

Ao iniciar os cuidados destinados à manutenção da integridade da pele, a avaliação estruturada do risco para o desenvolvimento de lesão por pressão deve ser realizada precocemente após admissão na unidade de terapia intensiva, sendo necessária a reavaliação quando houver mudança significativa no quadro clínico do paciente ${ }^{(6)}$.

Para que a avaliação do risco seja feita com qualidade em qualquer que seja a escala, o enfermeiro deve ser capaz de reconhecer os fatores de risco relacionados ao desenvolvimento de lesão por pressão, sendo os prioritários a mobilidade no leito, a perfusão e oxigenação, a situação nutricional deficiente, a exposição à umidade e à fricção e cisalhamento ${ }^{(15-18,21-23)}$. Portanto, o instrumento a ser utilizado deve ser capaz de apontar cada um desses fatores em sua avaliação.

As escalas de avaliação de risco geram escores que classificam o risco do paciente em desenvolver lesões por pressão, a forma de classificação varia entre as escalas. As ações e medidas a serem adotadas após classificação do risco do paciente devem ser claras e evidenciadas a fim de que a equipe saiba como lidar 
com situações de baixo risco para o desenvolvimento de lesões por pressão e situações de alto risco, em que a abordagem preventiva deverá ser precoce e de qualidade ${ }^{(6)}$.

A avaliação da pele deverá diagnosticar regiões hiperemiadas, risco ou formação de lesões causadas por dispositivos médicos, lesões já existentes, qualidade da cicatrização de lesões e piora/melhora da qualidade da pele após início das intervenções preventivas. A periodicidade da avaliação é amplamente discutida entre alguns das escala e varia muito entre as referências, sendo sugerida diariamente, semanalmente e, por vezes, não ficando clara a periodicidade $\mathrm{e}^{(11,13,17-18)}$. Foi possível observar que a periodicidade é escolhida de acordo com o risco do paciente. Logo, pacientes em alto risco devem ser reavaliados com maior brevidade possível $^{(15,17-18,21-23)}$.

A importância da documentação detalhada da avaliação da pele foi citada em algumas escalas, sendo discutida a importância do registro de qualidade para que possa favorecer o cuidado continuado e a percepção de melhora/piora nos resultados da avaliação ${ }^{(11,15,17,21)}$.

Em uma unidade neurológica nos EUA, os administradores de enfermagem e seus funcionários criaram uma equipe especializada de avaliação de feridas para monitorar e prevenir lesões de pele adquiridas no hospital. Os profissionais passaram por processo formativo com guia de referência para as principais ações e técnicas visando diagnosticar, prevenir e tratar lesões ${ }^{(17)}$. Outros estudos apontaram a importância do treinamento e da educação continuada dos enfermeiros que realizarão a avaliação da pele e aplicação de medidas preventivas( ${ }^{(12,14,21-22)}$.

A avaliação da pele será influenciada pela qualidade do exame físico realizado pelo enfermeiro, tornando tal ação fundamental para o profissional em unidade de terapia intensiva. A técnica propedêutica da inspeção será aliada direta na busca por sinais e sintomas na pele e reconhecimento de situações que podem representar riscos para a manutenção da integridade da pele.
Os estudos avaliados recomendam como principais cuidados preventivos para o surgimento de lesões evitar o posicionamento sobre áreas corporais ruborizadas, consideradas de maior risco, manutenção contínua de pele limpa e seca usando produtos de $\mathrm{pH}$ equilibrado com o da pele; não massagear as regiões já ruborizadas/hiperemiadas; desenvolver plano de cuidados específicos para pacientes com problemas de incontinência realizando limpeza imediata após cada episódio; proteger a pele da exposição à umidade pelo uso de produtos barreira, reduzindo, assim, o risco de danos; e, por fim, considerando a utilização de emolientes para hidratação da pele seca e risco de dano da pele $\mathrm{e}^{(15,17-18,21-23)}$.

Cabe ressaltar que, mesmo sendo possível encontrar nos estudos indicações para que se atue ativamente na prevenção de lesões por pressão relacionada à incontinência, poucas escalas trazem essa variável. A importância de saber agir perante as incontinências se dá ao fato de que a exposição à umidade causa alterações no $\mathrm{pH}$ da pele, fisiologicamente ácido, que se torna básico, fragilizando a pele e deixando-a mais suscetível aos efeitos de ventos de fricção e cisalhamento $^{(14,18-19)}$.

No que diz respeito ao elemento constitutivo massagem, os estudos demonstram que, quando feita em regiões hiperemiadas, pode causar o rompimento de vasos nos tecidos subjacentes, além de doloroso, esfregar a pele pode causar lesão tecidual ou reação inflamatória, especialmente nos idosos que possuem tecidos frágeis ${ }^{(8,18-19)}$.

0 uso de creme barreira foi citado nos estudos podendo ser utilizado nas regiões de proeminências ósseas e na pele perianal e perineal, que serão afetadas em episódios de incontinência. Cabe ressaltar que a aplicação não deve ser feita em técnica de massagem, tendo em vista os prejuízos já citados ${ }^{(15,18-19)}$.

Foi possível evidenciar na análise das escalas a indicação de utilização de ácidos graxos essenciais como produto emoliente para a proteção e hidratação da epiderme ${ }^{(14,17)}$. Existem diversos tipos de ácidos graxos, mas, sobre feridas, o ácido linoleico e o lino- 
lênico são os mais evidenciados ${ }^{(15,18-19)}$. Estes formam uma barreira de proteção, impedindo maceração, sendo importantes agentes nos processos de inflamação celular, proporcionando alívio após a primeira aplicação e nutrição celular local, possuindo capacidade de regeneração dos tecidos ${ }^{(15,18-19)}$.

Evidenciou-se a nutrição como aspecto importante para os cuidados preventivos relacionados à lesão por pressão. 0 rastreio nutricional é o processo utilizado para identificar os pacientes que necessitam de avaliação completa do seu estado nutricional devido às características que os colocam em potencial risco nutricional. 0 enfermeiro qualificado poderá realizar o rastreio do estado nutricional, devendo ser realizado no momento da admissão na instituição de saúde ou durante a primeira consulta ambulatorial. Os indivíduos com risco para desnutrição ou estado de deficit nutricional identificado devem ser encaminhados para os cuidados de nutricionistas ou equipes nutricionais multidisciplinares ${ }^{(13,15,18,20)}$.

Um dos estudos analisados trouxe a possibilidade de implantação de uma escala de prevenção de lesão por pressão específica para cuidados nutricionais ${ }^{(20)}$. As evidências apontaram como aspectos importantes a manutenção de ingestão calórica diária conforme necessidades corporais individuais de cada paciente. Além disso, considera-se importante a avaliação da ingestão proteica, enriquecimento da dieta com vitaminas e sais minerais, além de escolher a forma ideal de administração da dieta (via oral, enteral) ${ }^{(13,15,18,20)}$. Avaliação do estado nutricional deve estar presente logo na admissão do paciente em unidade de terapia intensiva e pode ser realizada com auxílio de instrumentos para avaliação.

É fundamental aprazar planos de reposicionamento em que constem a frequência e a duração da alternância das posições. Entre as escalas analisadas, o período de alternância variou entre 2-4 horas. A periodicidade deve considerar os seguintes aspetos do indivíduo: tolerância tecidual, nível de atividade e mobilidade, quadro clínico geral, objetivos do tratamento, estado da pele e conforto ${ }^{(14,15,18,23)}$.
Na escala implementada em uma unidade cardiológica que recebe paciente em pós-operatório de cirurgias cardíacas, as práticas de mudança de posição tiveram que ser revistas por conta da instabilidade hemodinâmica dos pacientes, mudanças de decúbito frequentes e abruptas causavam edema por má perfusão, hemorragia refratária e arritmias malignas. A técnica usada pelos enfermeiros da unidade sugeriu o giro em 10 graus por 10 minutos e visualização atenta de parâmetros do paciente, podendo ser adicionados mais 10 graus conforme tolerado ${ }^{(21)}$.

0 reposicionamento é um dos aspectos que mais sofre modificações com o desenvolvimento de novos estudos. 0 tempo de reposicionamento vem diminuindo cada vez, demonstrando, assim, um fator importante na prevenção de lesões, devendo ser o foco de campanhas com a equipe de enfermagem por ser uma técnica de baixo custo, que exige pouca expertise e age aliviando a pressão sobre superfícies da pele, aspecto diretamente relacionado à gênese de lesões.

As superfícies de apoio são dispositivos de redistribuição da pressão desenvolvidos para gerenciar cargas teciduais, o microclima e funções terapêuticas (ou seja, colchões, sistema integrado na cama, substituição de colchões, colchões de sobreposição, almofadas de assento ou sobreposições de almofadas de assento) ${ }^{(14-15)}$.

Sua escolha deve ser individualizada, dependendo das necessidades de redistribuição da pressão e de outras funções terapêuticas do paciente. As recomendações do fabricante devem ser respeitadas. As normas vão igualmente ao encontro das necessidades dos fabricantes enquanto guias de desenvolvimento dos produtos e reforço da garantia de qualidade ${ }^{(14-15)}$.

Entre os estudos, destacou-se a escala adotada por uma unidade cardiológica nos EUA. Para reduzir a incidência de lesões por pressão, a equipe da unidade de terapia intensiva decidiu, além de fornecer cuidados de enfermagem preventiva, usar uma cama de terapia de fluido a ar para pacientes em pós-operatório de cirurgia cardiológica previamente selecionados. 0 
leito foi escolhido porque atingiu baixas pressões de interface entre a superfície e o paciente, proporcionando imersão e envolvimento máximos. 0 paciente flutua em uma cobertura que encerra contas de silício fluidizadas, minimizando o cisalhamento e fricção. A umidade flui pelo leito, minimizando também a maceração da pele ${ }^{(16)}$.

O uso do colchão de espuma foi destacado em dois estudos ${ }^{(14-15)}$. Outros estudos citaram o uso de colchões para redistribuição da pressão, porém não especificaram qual o tipo utilizado. É importante que as superfícies utilizadas para diminuição da pressão passem por revisão periódica da sua qualidade, como os colchões de espuma, por exemplo, ao ser cessada a pressão, devem retornar à sua conformação original. Os colchões de espuma reativa de alta especificidade são mais aconselhados em relação aos que não sejam de alta especificidade $\mathrm{e}^{(11,13,19)}$.

Outros cuidados sugerem que deve ser avaliado se os colchões de gel têm quantidade suficiente de gel na sua totalidade e não há áreas onde o gel foi removido. 0 colchão de ar alternante enche e esvazia corretamente. É destacado que não se deve utilizar colchões de ar nem colchões de sobreposição de pressão alternada com células de diâmetro menor do que dez centímetros, pois não conseguem insuflar ar suficientemente capaz de assegurar o alívio de pressão sobre as células que se encontram desinfladas ${ }^{(11,13,19)}$.

Poucas escalas evidenciaram cuidados com dis-

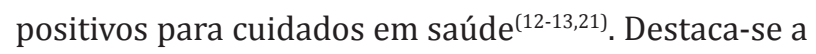
utilizada em unidade cardiológica, que descreveu os cuidados padronizados para cada tipo de dispositivo utilizado na unidade (cateteres centrais, cateter para aferição de pressão arterial invasiva, dispositivo mecânico de assistência circulatória, tubo endotraqueal, traqueostomia, sondas de alimentação) ${ }^{(21-23)}$.

Todos os pacientes em uso de algum dispositivo médico devem ser considerados como em risco para o desenvolvimento de lesões associadas ao dispositivo. Examinar a pele sob e ao redor dos dispositivos para cuidado em saúde, pelo menos duas vezes por dia, para identificar sinais de lesão no tecido adjacente é um cuidado que deve ser prontamente instituído ${ }^{(21-23)}$. Para cateteres de inserção em veia jugular interna ou femoral, indica-se o uso de filmes transparentes para a proteção do pescoço, orelha e virilha. Visando evitar o desenvolvimento de lesões relacionadas à transmissão para um dispositivo de assistência ventricular esquerdo, a transmissão deve ser estabilizada na pele do paciente com uma âncora de tubulação (fixador de cateteres). A pele é avaliada sob a âncora a cada cinco dias, com substituição da âncora, conforme necessário ${ }^{(21)}$.

Para os cuidados com a traqueostomia, as suturas não são mais usadas rotineiramente para garantir fixação de traqueostomia percutânea, sendo substituídas por velcro. Reduzir o uso de suturas com pacientes de traqueostomia percutânea de rotina permitiu que os enfermeiros conseguissem alívio de pressão na região de inserção da traqueostomia ${ }^{(21)}$.

As lesões da mucosa do tubo endotraqueal incluíram a maioria das lesões relacionadas aos dispositivos, levando a equipe de terapia intensiva a adicionar vigilância do reposicionamento do tubo em suas rondas. As recomendações de melhores práticas para o gerenciamento do tubo incluem a rotação a cada 24 horas quando a fita é usada como método de segurança. Abordagem semelhante foi adotada com as sondas de alimentação, trocam-se as sondas nasogástricas de posição a cada 24 horas para prevenir lesões na mucosa. 0 reposicionamento requer a inspeção, remoção e aplicação da data e hora na fita adesiva de segurança que fixa a sonda ${ }^{(21)}$.

A análise global dos achados e as discussões suscitadas por eles demonstram que a prevenção de lesões por pressão e os cuidados de enfermagem estão estreitamente relacionados. Para apropriar-se desse campo, é importante que seja do interesse de pesquisadores da enfermagem a busca por evidências cada vez melhores como forma de basear a prática em evidências e ampliar a qualidade do cuidado realizado.

Por fim, cabe ressaltar que a escala de Braden e suas versões adaptadas foram as mais utilizadas para 
prevenção de lesões por pressão em unidades de terapia intensiva, o que reforça a hegemonia do uso dessa escala em todo ambiente hospitalar. Não obstante, faz-se necessária a realização de mais revisões, inclusive sistemática com metanálise para que se possa gerar evidência que comprove o efeito do uso dessa escala em relação a desfechos graves.

\section{Conclusão}

Entre os elementos constitutivos avaliados pelas escalas, destacam-se a avaliação do risco para desenvolvimento de lesão por pressão com uso de instrumento validado; a avaliação da pele seguindo periodicidade preestabelecida e registros de qualidade para garantir a continuidade dos cuidados; cuidados com a pele, com destaque para a prevenção de excesso de umidade e uso de produtos para garantir a hidratação da pele e sua proteção. Os elementos constitutivos evidenciados nas escalas avaliadas contribuem para prevenção de lesão por pressão em unidades de terapia intensiva.

Contudo, ficou evidenciado que prevenção de lesões relacionada às incontinências, avaliação nutricional, intervenções nutricionais com o objetivo de prevenir lesões e os cuidados relacionados a dispositivos médicos são elementos constitutivos do cuidado de enfermagem pouco explorados ou até ausentes nas escalas avaliadas. Esses quatro pontos devem ser vistos com urgência, tendo vista sua importância na prevenção de lesões evidenciada na literatura.

\section{Colaborações}

Almeida ILS, Garces TS e Oliveira GYM contribuíram na concepção e projeto, análise e interpretação dos dados, redação do artigo e revisão crítica relevante do conteúdo intelectual. Moreira TMM contribuiu na redação do artigo, revisão crítica relevante do conteúdo intelectual e aprovação final da versão a ser publicada.

\section{Referências}

1. Moraes J, Borges E, Lisboa C, Cordeiro D, Rosa E, Rocha N. Conceito e classificação de lesão por pressão: atualização do National Pressure Ulcer Advisory Panel. Enferm Cent 0 Min 2016; 6(2):2292-306. doi: http://dx.doi.org/10.19175/ recom.v6i2.1423

2. Silva R, Figueiredo N, Meireles I, Costa M, Silva C. Feridas: fundamentos e atualizações em enfermagem. São Caetano do Sul: Yendis; 2013.

3. Barbosa T, Beccaria L, Poletti N. Pressure ulcer risk assessment in intensive care unit: preventive nursing care. Rev Enferm UERJ [Internet]. 2014 [cited Jun 15, 2019];22(3):353-8. Available from: https://www.e-publicacoes.uerj.br/index.php/ enfermagemuerj/article/view/13724

4. Inoue $\mathrm{K}$, Matsuda L. Cost-effectiveness of two types of dressing for prevention of pressure ulcer. Acta Paul Enferm. 2015; 28(5):415-19. doi: http:// dx.doi.org/10.1590/1982-0194201500070

5. Lima A, Castilho V. Body mobilization for prevention of pressure ulcers: direct labor costs. Rev Bras Enferm. 2015; 68(5):930-6. doi: http://dx. doi.org/10.1590/0034-7167.2015680523i

6. National Pressure Ulcer Advisory Panel, European Pressure Ulcer Advisory Panel and Pan Pacific Pressure Injury Alliance. Prevention and treatment of pressure ulcers: quick reference guide. Australia: Cambridge Media; 2014.

7. Whittemore R, Knafl K. The integrative review: updated methodology. J Adv Nurs. 2005; 52(5):54653.doi:http://dx.doi.org/10.1111/j.13652648.2005.03621.x

8. Mendes K, Silveira R, Galvão C. Revisão integrativa: método de pesquisa para a incorporação de evidências na saúde e na enfermagem. Texto Contexto Enferm. 2008; 17(4):758-64. doi: http://dx. doi.org/10.1590/S0104-07072008000400018.

9. Mendes K, Silveira R, Galvão C. Use of the bibliographic reference manager in the selection of primary studies in integrative reviews. Texto Contexto Enferm. 2019; 28:e20170204. doi: http:// dx.doi.org/10.1590/1980-265x-tce-2017-0204 
10. Fineout-Overholt E, Stillwell SB. Asking compelling, clinical questions. In: Melnyk BM, Fineout-Overholt E. Evidence-based practice in nursing \& healthcare: a guide to best practice. Philadelphia: Lippincot Williams \& Wilkins; 2011.

11. Louro M, Ferreira M, Póvoa P. Avaliação de protocolo de prevenção e tratamento de úlceras de pressão. Rev Bras Ter Intensiva. 2007; 19(3):337-41. doi: http://dx.doi.org/10.1590/ S0103-507X2007000300012

12. Elliott R, McKinley S, Fox V. Quality improvement program to reduce the prevalence of pressure ulcers in an intensive care unit. Am J Crit Care [Internet]. 2008 [cited Jun 15, 2019];17(4):32834. Available from: https://www.ncbi.nlm.nih. gov/pubmed/18593831

13. Pasek T, Geyser A, Sidoni M, Harris P, Warner J, Spence A, et al. Skin care team in the pediatric intensive care unit: a model for excellence. Am J Crit Care [Internet]. 2008 [cited Jun 15, 2019];28(2):125-35. Available from: http://ccn. aacnjournals.org/content/28/2/125.full

14. Coloma S, Ayllon-Garrido N, Latorre-García K. Evolution of pressure ulcers incidence after the improvement of a prevention protocol in intensive care. GEROKOMOS [Internet]. 2008 [cited Jun 11, 2019];19(4):207-12. Available from: http://scielo.isciii.es/scielo.php?script=sci_ arttext\&pid=S1134-928X2008000400007

15. Bereta R, Zborowski I, Simão C, Anselmo A, Ribeiro S, Magnani L. Protocolo assistencial para prevenção de úlcera por pressão em clientes críticos. CuidArte Enferm Internet]. 2010 [citado 2019 jun. 19]; 4(2):80-6. Disponível em: http:// www.fundacaopadrealbino.org.br/facfipa/ner/ pdf/CuidArte $\% 20$ Enfermagem $\% 20$ v. $\% 204 \% 20$ n.\%202\%20jul.dez\%202010.pdf
16. Jackson M, McKenney T, Drumm J, Merrick B, LeMaster T, VanGilder C. Pressure Ulcer Prevention in High-Risk Postoperative Cardiovascular Patients. Crit Care Nurse. 2011; 31(4):44-53. doi: http://dx.doi.org/10.4037/ccn2011830

17. Epstein N, McGuinness J, Persaud-Roberts S, Marra $S$, Ramos J, Toscano D, etal. How to reduce hospitalacquired pressure ulcers on a neuroscience unit with a skin and wound assessment team. Surg Neurol Int. 2012; 3(1):138. doi: http://dx.doi. org/10.4103/2152-7806.103645

18. Rogenski N, Kurcgant P. The incidence of pressure ulcers after the implementation of a prevention protocol. Rev Latino-Am Enfermagem. 2012; 20(2):333-9. doi: http://dx.doi.org/10.1590/ S0104-11692012000200016

19. Visscher M, King A, Nie A, Schaffer P, Taylor T, Pruitt D, et al. A Quality-Improvement Collaborative Project to Reduce Pressure Ulcers in PICUs. Pediatrics. 2013; 131(6):e1950-e1960. doi: http://dx.doi.org/10.1542/peds.2012-1626

20. Zepeda E, Martín C. Giving a nutritional FAST HUG in the Intensive Care Unit. Nutr Hosp. 2015; 31(5):2212-9. doi: http://dx.doi.org/10.3305/ nh.2015.31.5.8668

21. Cooper D, Jones S, Currie L. Against all odds: preventing pressure ulcers in high-risk cardiac surgery patients. Crit Care Nurse. 2015; 35(5):7682. doi: http://dx.doi.org/10.4037/ccn2015434

22. Swafford K, Culpepper R, Dunn C. Use of a comprehensive program to reduce the incidence of hospital-acquired pressure ulcers in an intensive care unit. Am J Crit Care. 2016; 25(2):152-5. doi: http://dx.doi.org/10.4037/ajcc2016963

23. Mendonça P, Loureiro M, Frota O, Souza A. Prevention of pressure injuries: actions prescribed by intensive care unit nurses. Texto Contexto Enferm. 2018; 27(4):e4610017. doi: http://dx.doi. org/10.1590/0104-07072018004610017

\section{(cc) BY}

Este é um artigo de acesso aberto distribuído sob os termos da Licença Creative Commons 